\title{
Epidemiological profile of tinea capitis in São Paulo City*
}

\author{
John Verrinder Veasey ${ }^{1}$ \\ Silvia Assumpção Soutto Mayor ${ }^{1}$ \\ Laura Hitomi Muramatu
}

\author{
Barbara Arruda Fraletti Miguel ${ }^{1}$ \\ Clarisse Zaitz ${ }^{1}$ \\ Juliane Agarinakamura Serrano ${ }^{3}$
}

DOI: http://dx.doi.org/10.1590/abd1806-4841.20175463

\begin{abstract}
Tinea capitis is the most common fungal infection in children. The identification of the etiologic agent helps clinicians make their therapeutic choice. Studies conducted in different countries show a changing pattern of the main etiological agents according to their regions. We performed a retrospective study in the tertiary public service in São Paulo, analyzing the isolated etiological agents in patients with tinea capitis from March 2013 to May 2015. Microsporum canis was the main agent $(56.6 \%)$, followed by Trichophyton tonsurans (36.6\%). Despite recent migratory movements in the city, we observed no change in the causative agent of tinea capitis.
\end{abstract}

Keywords: Epidemiology; Microsporum; Mycoses; Tinea capitis; Trichophyton

Dermatophytoses are superficial cutaneous mycoses caused by dermatophytes that can also be called tinea. These fungi use keratin of the skin, hair, or nails as a substrate. The species that live in the soil occasionally infect humans and are called geophilic; the zoophilic species parasitize animals and rarely infect humans; anthropophilic species preferably parasitize humans. Tinea capitis usually affects children under 12 years of age and peak incidence is observed at 3-7 years of age. It may be clinically classified as microsporic (characterized by a single lesion caused preferably by zoophilic and geophilic dermatophytes) or trichophytic (characterized by multiple lesions and usually caused by anthropophilic dermatophytes). ${ }^{1,2,3}$ The frequency of etiologic agents varies according to the region and study period. In Brazil, Microsporum canis is the most common causative agent in the South and Southeast regions, while Trichophyton tonsurans is more common in the North and Northeast regions. ${ }^{1}$

In recent years, a change has been described in the incidence of major causative agents of tinea capitis in Europe and the United States. ${ }^{4}$ Zoophilic agents were of great importance in these regions and represented the main causal agents of fungal infections of the scalp; currently, anthropophilic fungi are the most common species isolated from tinea capitis, especially in large cities with immigrants coming from Africa and the Caribbean. ${ }^{5}$

We conducted a retrospective study at our dermatology clinic in the city of São Paulo by reviewing the results of mycological and culture tests performed in patients with the diagnosis of tinea capitis from March 2013 to May 2015.
All cases with clinical suspicion underwent direct mycological examination and fungal culture. Sixty-seven patients suspected of tinea capitis were treated. Of them, all had hyaline septate hyphae revealed on direct microscopic examination. However, only 30 cultures isolated the etiologic agent (37 were negative). Among the identified agents, it was observed a predominance of Microsporum canis (17 cultures: $56.6 \%$ ), followed by Trichophyton tonsurans (11 cultures: $36.6 \%$ ), Microsporum gypseum (1 culture: $3.3 \%$ ), and Trichophyton mentagrophytes (1 culture: $3.3 \%$ ). The incidence was higher in males ( $64.17 \%$ of the 67 cases), and the mean age was 8.13 years, with a standard deviation of 8.31 .

In Europe, there is a change towards tinea capitis infections by anthropophilic dermatophytes in urban areas. The largest increase of this agent has been observed in the United Kingdom ( $T$. tonsurans) and France (T. soudanense and M. audouinii). ${ }^{6}$ In Eastern Europe, there is a dominance of tinea capitis caused by zoophilic fungi (M. canis). The effects of the civil war in some European countries in the 1980s and 1990s resulted in low standards of medical care and M. canis outbreaks, possibly related to the increase in the number of wild pets. The end of the war, with the subsequent improvement of living standards, led to a reduction in zoophilic infections and to an increase in anthropophilic infections. ${ }^{7}$ In rural areas of Northeastern France, for example, the main causative agent of dermatophytosis are zoophilic fungi like M. canis, T. mentagrophytes, and T. verrucosum. However, in urban areas in Paris, for example, there is a swift increase of dermatophytoses caused by anthropophilic fungi, and the main agents are T. tonsurans, T. soudanense, and

Received on 03.12.2015

Approved by the Advisory Board and accepted for publication on 08.05.2016

* Work performed at Dermatology Clinic at Hospital Irmandade da Santa Casa de Misericórdia de São Paulo - São Paulo (SP), Brazil.

Financial support: none.

Conflict of interest: none.

Dermatology Clinic at Irmandade da Santa Casa de Misericórdia de São Paulo - São Paulo (SP), Brazil.

Mycology Laboratory at Irmandade da Santa Casa de Misericórdia de São Paulo - São Paulo (SP), Brazil.

School of Medical Sciences at Santa Casa de São Paulo (FCMSCSP) - São Paulo (SP), Brazil.

(C)2017 by Anais Brasileiros de Dermatologia 
M. audouinii. This pattern of infection appears to be linked to immigration from sub-Saharan Africa or the Caribbean. ${ }^{6}$

In her Master's thesis, Mayor evaluated 402 cultures performed from children up to 10 years of age in the city of São Paulo between January 1999 and December 2003. The following figures were obtained: M. canis (45.5\%), T. tonsurans (40.3\%), M. gypseum (5.5\%), T. mentagrophytes $(0.5 \%)$, and T. rubrum $(0.3 \%) .{ }^{8}$ In the present study, M. canis remained the dominant cause of tinea capitis, followed by $T$. tonsurans (and we highlight the distance between the frequency of the first over the second).

In the epidemiological study by Marques et al. at the Faculty of Medicine in Botucatu (SP), a service that traditionally receives patients from the rural area, the most isolated dermatophyte species was M. canis in $88.2 \%$ of the 364 species isolated from tinea capitis. This shows, from a sanitary point of view, the importance of isolating the zoophilic or anthropophilic agent. ${ }^{9}$

\section{REFERENCES}

1. Zaitz C, Marques SA, Ruiz LRB, Framil VMS. Compêndio de Micologia Médica. 2. ed. Rio de Janeiro: Guanabara Koogan; 2010. p.157-159.

2. Möhrenschlager M, Bruckbauer H, Seidl HP, Ring J, Hofmann H. Prevalence of asymptomatic carriers and cases of tinea capitis in five to six-year old preschool children from Augsburg, Germany: results from the MIRIAM study. Pediatr Infect Dis J. 2005;24:749-50.

3. Sharma V, Hall JC, Knapp JF, Sarai S, Galloway D, Babel DE. Scalp colonization by Trichophyton tonsurans in an urban pediatric clinic - asymptomatic carrier state. Arch Dermatol. 1988;124:1511-3.

4. Hay RJ, Robles W, Midgley G, Moore MK; European Confederation of Medical Mycology Working Party on Tinea Capitis. Tinea capitis in Europe: new perspective on an old problem. J Eur Acad Dermatol Venereol. 2001;15:229-33.

5. Binder B, Lackner HK, Poessl BD, Propst E, Weger W, Smolle J, et al. Prevalence of tinea capitis in Southeastern Austria between 1985 and 2008: up-to-date picture of the current situation. Mycoses. 2011;54:243-7.

6. Ginter-Hanselmayer G, Weger W, Ilkit M, Smolle J. Epidemiology of tinea capitis in Europe: current state and changing patterns. Mycoses. 2007;50:6-13.

7. Foulet F, Cremer G, Bourdon-Lanoy E, Wolkenstein P, Chosidow 0, Bretagne S, et al. Frequency of plantar dermatophytosis. A retrospective study 2002. Ann Dermatol Venereol. 2007;134:343-5.

8. Soutto Mayor SA. Dermatofitoses na infância (zero a dez anos de idade): Análise de cinco anos de atendimento na clínica de dermatologia da Santa Casa de São Paulo [dissertação], São Paulo ( SP), Faculdade de Ciências Médicas da Santa Casa de São Paulo; 2005.

9. Marques SA, Camargo RMP, Fares AHG, Takashi RM, Stolf HO. Tinea capitis: epidemiologia e ecologia dos casos observados entre 1983 e 2003 na Faculdade de Medicina de Botucatu, Estado de São Paulo, Brasil. An Bras Dermatol. 2005;80:597-602.

10. Gupta AK, Drummond-Main C. Meta-analysis of randomized, controlled trials comparing particular doses of griseofulvin and terbinafine for the treatment of tinea capitis. Pediatr Dermatol. 2013;30:1-6.
A meta-analysis of randomized controlled trials showed better results in the treatment of tinea capitis caused by Microsporum $s p$. with griseofulvin compared with terbinafine, and the reverse for tinea capitis by Trichophyton $s p .{ }^{10}$ Therefore, the importance of isolating the causative agent is demonstrated by the superiority in efficacy of treatment depending on the agent. Furthermore, as most of the cultures yielded negative results, the need to know the most frequent etiological agent in the population of interest is paramount so that the empiric treatment, if necessary, is more effective.

Epidemiological studies similar to the present one should be performed to observe a possible change in the main causative agent of tinea capitis in the coming years since the most prevalent infecting species undergo variations depending on study time, country, and regions within a country, depending on environmental factors, ethnic group, socioeconomic status, and population density. ${ }^{9}$ Therefore, the adoption of appropriate preventive and therapeutic measures could be taken based on the infectious agents.

How to cite this article: Veasey JV, Miguel BAF, Mayor SAS, Zaitz C, Muramatu LH, Serrano JA. Epidemiological profile of tinea capitis in São Paulo City. An Bras Dermatol. 2017;92(2):283-4. 